\title{
An Analog/Digital Baseband Processor Design of a UWB Channelized Receiver for Transmitted Reference Signals
}

\author{
LEI FENG AND WON NAMGOONG \\ Department of Electrical Engineering, University of Southern California \\ e-mail: $\{$ leifeng; namgoong $\} @ u s c . e d u$
}

\begin{abstract}
The frequency channelized receiver enables the use of practical analog-to-digital converters (ADC) to digitize ultra-wideband (UWB) signals. The design issues of the analog and digital baseband processor for the channelized receiver in a UWB transmitted reference (TR) system are investigated. In the analog part, the receiver performance is shown to be weakly dependent on the analog filter bandwidth, the filter order, and the ADC oversampling ratio assuming white input noise. In the digital part, the coarse acquisition performance is shown to be significantly better in a channelized receiver than in a fullband receiver. The implementation issues for fine synchronization and correlation window length are also studied.
\end{abstract}

Keywords: Ultra-wideband, transmitted reference, channelized receiver, analog-to-digital converter, coarse acquisition, synchronization

\section{Introduction}

Ultra-wideband (UWB) impulse radio, which operates by transmitting very short pulses with bandwidth on the order of several gigahertz, enjoys numerous advantages compared to narrowband systems including robustness to multipath fading and potential for achieving high capacity. Its very wide bandwidth, however, poses several new challenges to communication system designers [1]. Channel estimation and synchronization appear to be the two major problems. The difficulty of channel estimation arises from the dense multipath environment. Synchronization becomes a challenge because of the low duty cycle and the ultra-short nature of the transmitted pulse, both of which introduce non-negligible timing and frequency offset between pulses.

The above mentioned challenges motivate a receiver that digitizes the received UWB signals as early as possible so that advanced digital processing techniques can be applied. Since designing a single ADC to operate at the signal Nyquist rate is not practical, parallel ADC architectures with each ADC operating at a fraction of the Nyquist rate need to be employed. To sample at a fraction of the Nyquist rate, a frequency channelized receiver based on hybrid filter banks (i.e., continuoustime analysis filters and discrete-time synthesis filters) has been proposed in [2]. Among the advantages of the frequency channelized receivers compared to the more conventional time channelized (i.e., time-interleaved $\mathrm{ADC})$ receivers are the ease of designing the sample/hold circuitries, greater robustness to jitter/phase noise, and reduced ADC dynamic range requirements. The main drawback of the frequency channelized receiver, however, is the slow convergence speed, which can be problematic in time-varying UWB wireless environment [3].

Transmitted reference (TR) modulation schemes have been proposed because of the ease of channel estimation in a dense multipath environment and the low implementation complexity [4]. In one version of TR system, referred to as the averaged TR system 
[5], a fixed number of reference pulses precede data modulated pulses in each transmission block. The pulse rate is set so that there is no interference between consecutive pulses. Assuming that the channel is constant for a block duration, the reference pulses are averaged to estimate the channel response. This channel estimate is then used to recover the transmitted data by simply correlating with the data pulses. In the frequency channelized receiver, data detection in a TR system can be achieved by simply averaging the reference pulse responses in each of the subband channels independently and using them to correlate the data pulses [8].

In this paper, we address the analog/digital baseband design issues of the TR UWB channelized receiver. As designing bandpass filters with high center frequency is difficult, channelization is achieved using a bank of mixers operating at equally spaced frequencies and lowpass filters to decompose the analog input signal into subbands [2]. Ideally, the analog lowpass filter should satisfy the power complementary condition [8]. In practice, the filters are only approximately power complementary because of the difficulties in designing such filters and the uncertainties due to process and temperature variations. Hence, the receiver sensitivity to the filter order and the cutoff frequency are investigated. In addition, the ADC resolution and sampling requirements are studied. Mono-bit ADCs are of special interest because of its potential for low power design.

After digitization, the receiver must first acquire the initial synchronization parameters from the incoming pulses. Achieving acquisition is difficult in UWB systems due to the high pulse timing sensitivity and the large intervals between pulses [11][12]. Several fast acquisition schemes have been proposed [11][14][15][16]. In this paper, we show that the frequency channelized receiver naturally leads to fast acquisition in TR systems. Coarse acquisition in a TR system is achieved by performing a two-dimensional search for the pulse arrival time and the interval between consecutive pulses. The acquisition hypothesis variable is obtained by correlating between consecutive pulses in each subband, squaring to remove the phase uncertainty, then summing. For comparison, a full-band receiver, which is equivalent to a one-subband channelized receiver, is also considered. In the channelized receiver, the reduced bandwidth in each subband widens the correlation peak, allowing the search increment to be correspondingly increased. Consequently, the search space in the channelized receiver is reduced, resulting in a significantly faster acquisition time than that of a fullband receiver.

After the initial acquisition, the received signal goes through fine synchronization and detection. The timing synchronization is composed of an interpolator, a Mueller and Muller (M\&M) timing error detector (TED) and a numerically controlled oscillator (NCO) [10]. As the correlation window length is important in a TR system due to the "noise cross noise" term in the correlation [17], the length is adaptively adjusted. The window length effects are simulated.

The paper is organized as follows. Section 2 presents the UWB TR system model. The low-pass filter and $\mathrm{ADC}$ requirements of the channelizer are presented in Section 3. Coarse acquisition is discussed in Section 4, while Section 5 describes fine synchronization and detection. Section 6 draws conclusions.

\section{System Model}

To satisfy the FCC spectral mask, several pulse shapes have been proposed [6][7]. In this paper, we use the 5th-derivative of the Gaussian pulse

$$
p_{t r}(t)=A_{c}\left(-\frac{t^{5}}{\sqrt{2 \pi} \lambda^{5}}+\frac{t^{3}}{\sqrt{2 \pi} \lambda^{3}}-\frac{t}{\sqrt{2 \pi} \lambda}\right) e^{\frac{t^{2}}{2 \lambda^{2}}}
$$

where $A_{c}$ is a constant and $\lambda$ of $51 \mathrm{ps}$ to fill the FCC spectral mask in the $3.1-10.6 \mathrm{GHz}$ band. Since designing a receiver to support the entire UWB band may be difficult, we subsequently assume a more practical pulse with a bandwidth of $1.5 \mathrm{GHz}$ (3.1$4.6 \mathrm{GHz}$ ), which is obtained by filtering the pulse response in (1) with a fourth order Chebyshev bandpass filter. The resulting pulse waveform is shown in Fig. 1.

After passing through the multipath channel, the received pulse is $s(t)$. In each transmission block, the first $N_{r}$ pulses are reference pulses and the remaining $N_{d}$ are data pulses. To simplify the problem, we assume that the pulses are transmitted in fixed time intervals and that the interval is sufficiently large to prevent inter-pulse interference between consecutive pulses. The channel is assumed to be constant during a transmission block. A block of received signal is

$$
r(t)=\sum_{k=0}^{N_{r}-1} s(t-k T)+\sum_{k=N_{r}}^{N_{r}+N_{d}-1} a_{k} s(t-k T)+n(t)
$$




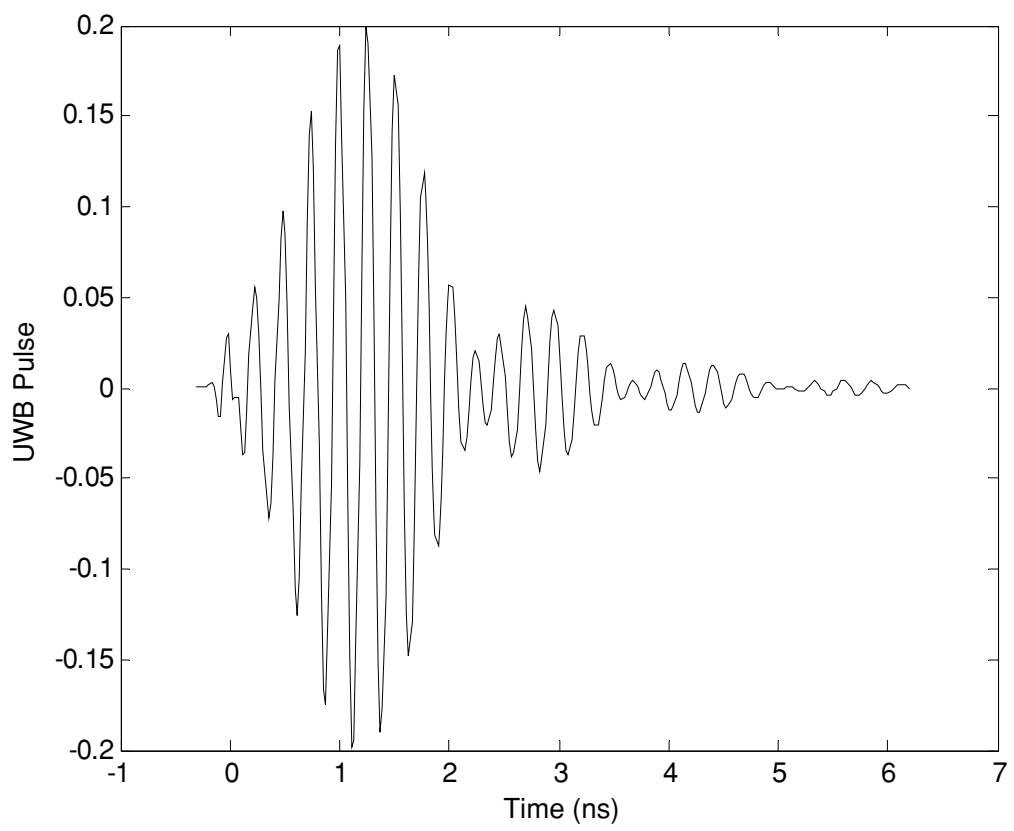

Figure 1. The transmitted pulse with $1.5 \mathrm{GHz}$ bandwidth.

where $a_{k}$ is the $k$ th transmitted antipodal symbol, $n(t)$ is the additive noise, and $T$ is the frame period (pulse repetition period). The channel estimation can be achieved by averaging the first $N_{r}$ pulses. The estimated pulse is then correlated with the other frame signals to detect the data. The short pulse duration indicates that the detection is very sensitive to timing offset.

\subsection{Full-Band Receiver}

Since the signal pulse energy falls within the 3.1$10.6 \mathrm{GHz}$ FCC spectral mask, the received signal is first mixed to the baseband so to relax the ADC sampling requirements. Denoting the mixer frequency as $f_{f}$, the $i$ th sample of the $k$ th pulse is

$$
\begin{aligned}
x_{k}[i] & =\left.e^{-j 2 \pi f_{f} t}\left(a_{k} s(t-k T)+n(t)\right)\right|_{t=k(T+\varepsilon)+i T_{s}} \\
& =a_{k} e^{-j 2 \pi f_{f} k T} s_{f u l l}\left(k \varepsilon+i T_{s}\right)+n_{f u l l, k}[i]
\end{aligned}
$$

where $T_{S}$ is the sampling period and $T+\varepsilon$ is the receiver's estimate of the pulse repetition period $T$. The fullband signal $s_{f u l l}(t)=s(t) e^{-j 2 \pi f_{f} t}$ and noise $n_{f u l l, k}[i]=\left.n(t) e^{-j 2 \pi f_{f} t}\right|_{t=k(T+\varepsilon)+i T_{s}}$.

\subsection{Channelized Receiver}

An $M$ subband channelizer is shown in Fig. 2. The received signal $r(t)$ is downconverted by a set of equally spaced mixers at frequencies $f_{0}, f_{1}, \ldots, f_{M-1}$, filtered by the lowpass filter $H(\omega)$, then sampled by ADCs operating at $1 / M$ of the full-band receiver sampling frequency. The channelized receiver ADC sampling frequency $f_{\text {sub }}$ is $1 /\left(M T_{s}\right)$. The $i$ th sample of the $k$ th pulse in the $m$ th subband can be shown to be

$$
\begin{aligned}
x_{k, m}[i]= & \left\{\left[a_{k} s(t-k T)+n(t)\right] e^{-j 2 \pi f_{m} t}\right\} \\
& \left.\otimes h(t)\right|_{t=k(T+\varepsilon)+i M T_{s}} \\
= & a_{k} e^{-j 2 \pi f_{m} k T} s_{m}\left(k \varepsilon+i M T_{s}\right)+n_{m, k}[i]
\end{aligned}
$$

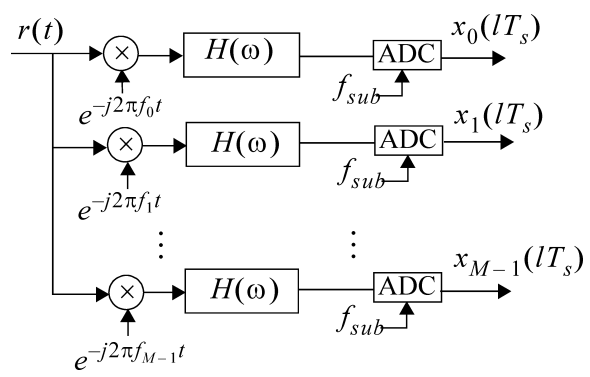

Figure 2. An $M$ subband UWB Channelizer. 
where $s_{m}(t)=\left[s(t) \otimes\left(h(t) e^{j 2 \pi f_{m} t}\right)\right] e^{-j 2 \pi f_{m} t}, n_{m, k}[i]=$ $\left.n(t) e^{-j \omega_{m} t} \otimes h(t)\right|_{t=k(T+\varepsilon)+i M T_{s}}$, and $h(t)$ is the impulse response of the lowpass filter.

\section{Channelizer}

In this section, the design requirements of the analog lowpass filter and the ADCs in the frequency channelized receiver are explored. The performance is analyzed assuming perfect synchronization.

\subsection{Analog Lowpass Filter}

In order to preserve the simple averaging and correlating detection structure of TR system, the subband filter should satisfy the power complementary condition and the sampling frequency should be high enough so to be free from aliasing [8]. The power complementary condition to be satisfied is

$$
\sum_{m=0}^{M-1}\left|H\left(\omega-2 \pi f_{m}\right)\right|^{2}=1
$$

In practice, the filters are only approximately power complementary because of the difficulties in designing such filters and the uncertainties due to process and temperature variations. Although perfect power complementary analysis filters can be designed by appropriately cascading analog and digital filters as described in [8], we consider approximately power complementary analog analysis filters with no digital filters to simplify the receiver complexity.

The analog lowpass filter order affects the amount of aliasing from sampling and the amount of overlap between adjacent subbands. To quantify the loss in performance when approximately power complementary analog analysis filters are used, the bit-error-rate (BER) curves of a 3-subband channelized receiver for TR with four training pulses and using the CM1 channel model provided in IEEE P802.15-02/368r5-SG3a are plotted in Fig. 3. The horizontal axis denotes the filter cutoff frequency normalized by half the difference between consecutive mixer frequencies. Higher filter orders are obtained by cascading identical first order filters. The received $E_{b} / N_{0}$, which is the ratio of the single pulse energy $\left(E_{b}\right)$ to the noise spectral density $\left(N_{0}\right)$, is $10 \mathrm{~dB}$. The signal bandwidth is $1.5 \mathrm{GHz}$ $(3.1-4.6 \mathrm{GHz})$ and the ADC sampling frequency is $2 / 3 \mathrm{GHz}$ with each mixer frequency separated by $500 \mathrm{MHz}$. In Fig. 3, the BER of a channelized receiver with power complementary analysis filter is plotted for reference.

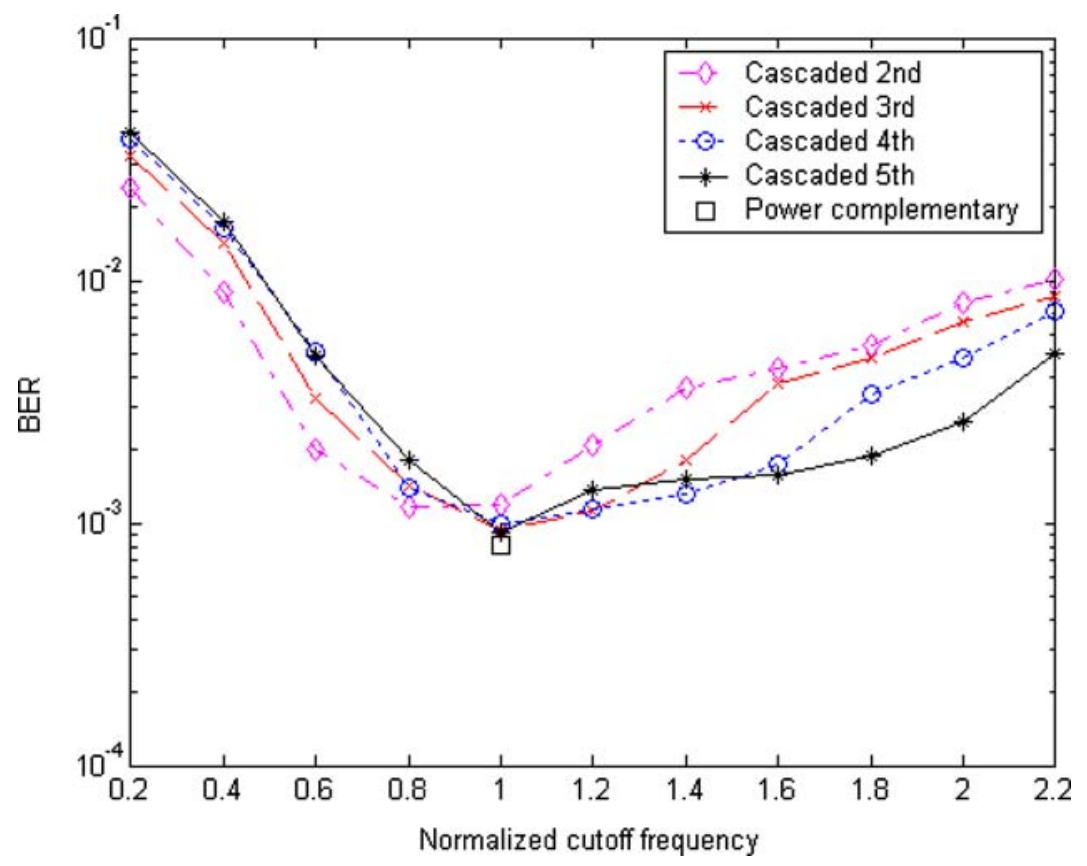

Figure 3. BER sensitivity to analysis filter cutoff frequency. 
As is clear from Fig. 3, the channelized receiver with approximately power complementary filters achieve the lowest BER (which is close to the ideal channelized receiver with power complementary filters) when the normalized cutoff frequency is approximately one. The minimum is also reasonably broad, suggesting that the channelizer is not very sensitive to slight variations in the filter response. The small difference among the minima implies that high order filters are not necessary when the input noise is white. In the presence of narrowband interference, however, sharp filters are needed to isolate the effects of the interferer as explained in [2]. In the remainder of this paper, we assume that a fourth order lowpass filter is used.

\subsection{Analog-to-Digital Converter}

The quantization requirements of the frequency channelized receiver is illustrated in Fig. 4. The BER curves for the TR system with four training pulses are plotted as a function of $E_{b} / N_{0}$ for different quantization levels. The ADC sampling frequency is $2 / 3 \mathrm{GHz}$, and the propagation channel model is assumed to be CM1. The BER of a full-band receiver, operating at $2 \mathrm{GHz}$, is shown for comparison. Except for the infinite-bit ADC receivers, the channelized receiver slightly outperforms the fullband receiver when the same ADC resolution is used. This improvement is due to the reduced quantization noise resulting from the reduction in signal power from the channelization process.

In Fig. 4, three bits are sufficient to achieve performance comparable to a channelized receiver with infinite-bit ADC resolution. When 1-bit ADCs are used, the loss in performance compared to an infinitebit channelized receiver is $2-3 \mathrm{~dB}$. Despite this loss in performance, the 1-bit ADC receiver is especially attractive because of the resulting hardware simplicity. A simple comparator can be used as an ADC and no automatic gain controller (AGC) is needed in each of the subband channel.

As increasing the sampling frequency reduces the effect of the quantization noise and aliasing from sampling, the performance of the channelized receiver can be improved by increasing the oversampling factor. In Fig. 5, the BER is plotted for different ADC sampling frequencies (1, 1.33 and 2), which are normalized by the mixer frequency difference of 500MHz. Both 1-bit and infinite-bit ADCs are simulated. The lowest normalized ADC sampling frequency that provides sufficient statistics of the received signal is one. While increasin the normalized sampling frequency beyond

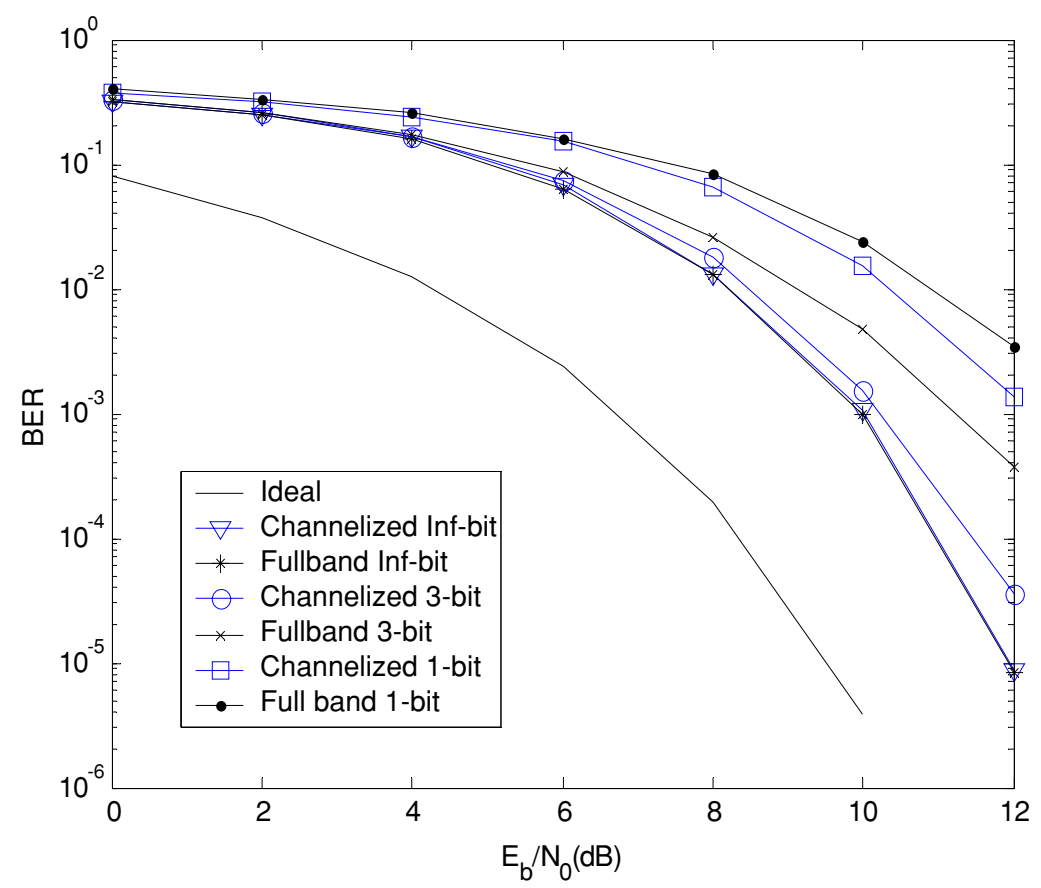

Figure 4. ADC resolution effects with 4 reference pulses. 


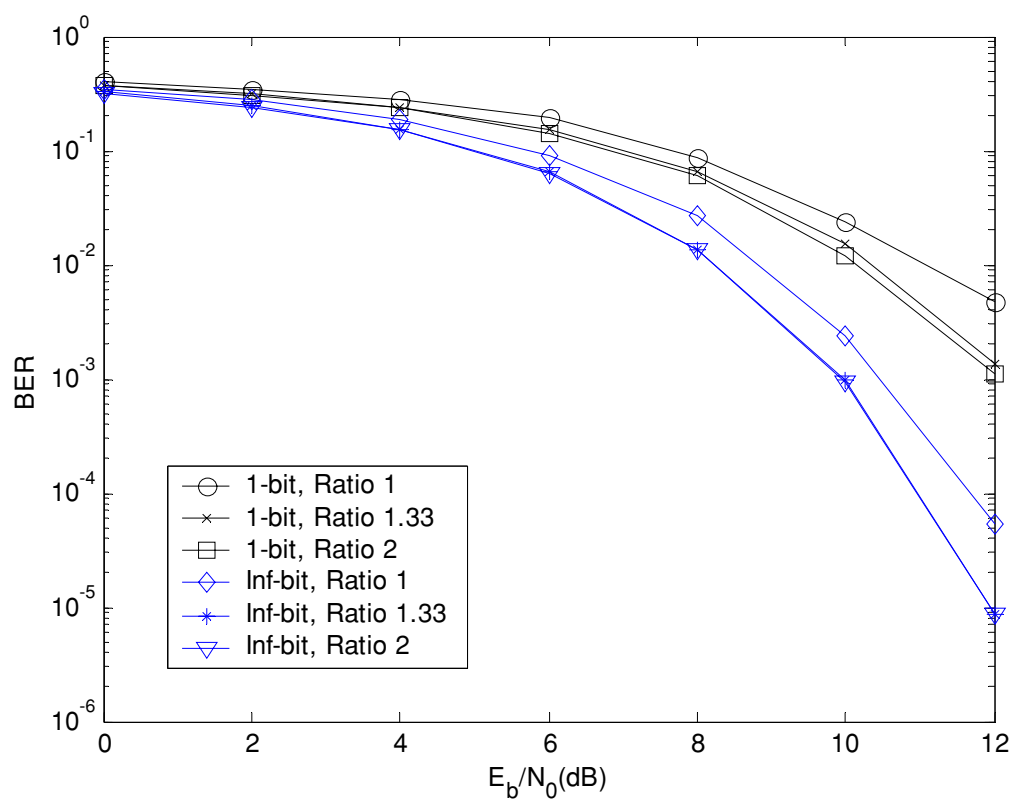

Figure 5. BER performance with different ADC sampling rates. ADCs sample signal at 1, 1.33 and 2 times the minimum sampling frequency.

1.33 for the infinite-bit ADC receiver shows no performance improvement, the 1-bit ADC receiver performance improves with increasing sampling frequency. The largest incremental improvement in performance, however, occurs when the normalized sampling frequency is slightly increased from one. Hence, we subsequently assume that the normalized ADC sampling frequency is 1.33 .

\section{Coarse Acquisition}

Since the transmitter and receiver clocks are not synchronized, the receiver must first perform coarse acquisition to estimate the pulse arrival time $\tau_{0}$ and the pulse repetition period $T$ before detection. To reduce the "noise cross noise" term, a time window is applied to each pulse in order to capture only the high signal-to-noise ratio part. The acquisition parameters
( $\tau_{0}$ and $T$ ) are shown in Fig. 6. $\tau_{0}$ is searched by sliding the two windows within the pulse repetition period $T$ while keeping their interval constant. Since the transmitter and receiver clocks are incommensurate, only a nominal value of $T$ is available at the receiver. The acquisition procedure of $T$ is to change the timing offset between two windows in the range corresponding to the clock uncertainty. For each tentative value of $\tau_{0}$, the pulse repetition period $(T)$ is searched from all possible time offsets by a certain step. The total number of search states is the multiplication of the numbers of two possible values.

In a full-band receiver, the coarse acquisition is achieved by first correlating the conjugate of the windowed $k$ th pulse with the windowed $(k+1)$ st pulse. The cross-correlation value is squared to remove any phase ambiguity. If the resulting hypothesis variable exceeds a certain threshold, the receiver enters the

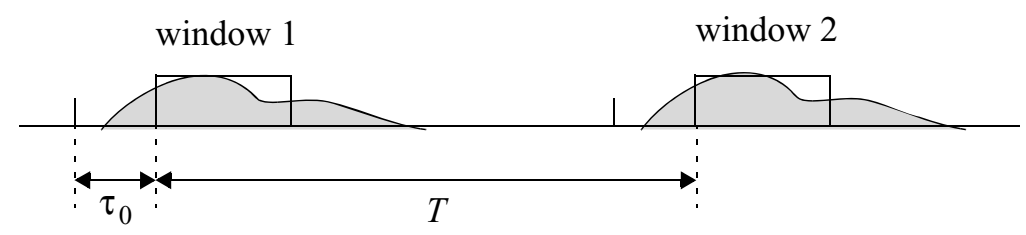

Figure 6. Acquisition parameters. 
fine synchronization mode. If the resulting value is below the threshold, the receiver changes the tentative values of $\tau_{0}$ and/or $T$ before continuing the search process.

The coarse acquisition process in the channelized receiver is similar to that in the full-band receiver. In each subband, the conjugate of the windowed $k$ th pulse is correlated with the windowed $(k+1)$ st pulse. The cross-correlation value in each subband is squared then summed. The estimates of both $\tau_{0}$ and $T$ are varied during the acquisition process. Coarse acquisition is completed when the resulting hypothesis variable exceeds a certain threshold. Compared to the full-band receiver, the squaring and summing operation in each subband reduces the probability of detection $P_{D}$ for a given $P_{F}$, which is the false alarm probability when signal is absent in both pulses. Despite this degradation, the overall coarse acquisition time improves because the frequency channelization process reduces the number of search bins. Since the reduced bandwidth in each subband channel widens the correlation peak by approximately the number of subbands $M$, the search increment (or bin) can be increased by $M$, resulting in a reduction of the search space by a factor of $M$. Assuming no degradation in the detection probability $\mathrm{P}_{\mathrm{D}}$, the acquisition time reduces by almost $M$ times. An example of the correlation for a fullband and 3-subband frequency channelized receiver is plotted in Fig. 7 as a function of the offset time assuming CM1 channel and no additive noise. Note that in addition to the widening of the correlation peak, multiple side-lobes emerge in the frequency channelized receiver, resulting in increased false alarm probabilities.

In this section, the acquisition performance of both the full-band and channelized receivers are analyzed. Serial-search algorithm is considered, that is, only one state is checked for a pulse period $T$. If successful acquisition is not reached at current state $l$, state $l+1$ is searched in the next period.

\subsection{Hypothesis Testing in a Full-Band Receiver}

The hypothesis testing is performed to decide whether correct acquisition is achieved when searching over all the states. The search states are labeled by $l=$ $0,1, \ldots, L_{1}$. The $l=0$ state corresponds to the correct acquisition state. For simplicity, we assume all pulses are unmodulated. At the $l$ th state, the samples in the windowed $k$ th pulse given in (3) can be represented as a $N_{w}$ dimension vector $e^{j \theta_{l, k}}\left(\mathbf{s}_{l, k}+\mathbf{n}_{l, k}\right)$, where $\mathbf{s}_{l, k}$ is the signal vector, $\mathbf{n}_{l, k}$ is the complex noise vector with covariance matrix $N_{0} \mathbf{I}\left(=2 \sigma^{2} \mathbf{I}\right)$ with $\mathbf{I}$ being an

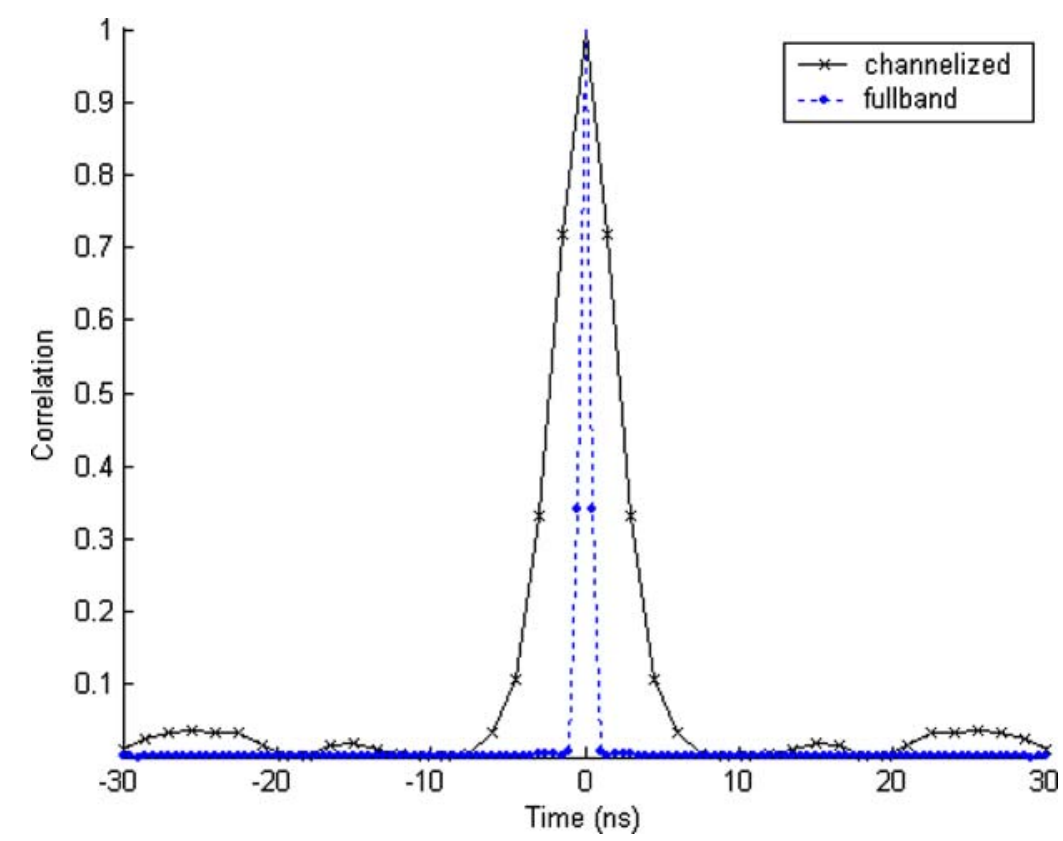

Figure 7. The hypothesis variables of a fullband signal and 3-subband channelized signals with respect to timing offset between two windows. 
identity matrix, and $\theta_{l, k}$ is the mixer phase for the $k$ th pulse at the $l$ th state. $\theta_{l, k}$ is time varying due to transmitter and receiver clock uncertainty. Correlating with the windowed $(k+1)$ st pulse, the resulting signal is

$$
\begin{aligned}
Y_{l}=e^{j\left(\theta_{l, k+1}-\theta_{l, k}\right)} & \left(\mathbf{s}_{l, k}^{H} \mathbf{s}_{l, k+1}+\mathbf{s}_{l, k}^{H} \mathbf{n}_{l, k+1}\right. \\
& \left.+\mathbf{n}_{l, k}^{H} \mathbf{s}_{l, k+1}+\mathbf{n}_{l, k}^{H} \mathbf{n}_{l, k+1}\right)
\end{aligned}
$$

where the superscript $H$ stands for conjugate transpose. The first term in the parenthesis on the right hand side of (6) is the signal component and the remaining terms are the noise components. The second and third terms are "signal cross noise" terms and are zero mean Gaussian noise. The fourth term, which is the "noise cross noise" term, is also approximately Gaussian by the Central Limit Theorem, since the noise in different pulses is independent. The magnitude of the mean of $Y_{l}$ is

$$
E_{l, f u l l}=\left|\mathbf{s}_{l, k}^{H} \mathbf{s}_{l, k+1}\right|
$$

and the variance of all the noise is

$$
\sigma_{l, f u l l}^{2}=\left|\mathbf{s}_{l, k}\right|^{2} \sigma^{2}+\left|\mathbf{s}_{l, k+1}\right|^{2} \sigma^{2}+N_{w} \sigma^{4}
$$

To remove the phase ambiguity, the hypothesis variable is obtained by squaring the correlated signal

$$
J_{l, \text { full }}=\left|Y_{l}\right|^{2}
$$

The hypothesis variable $J_{l, \text { full }}$ is then compared to a threshold to determine whether the signal is present. $J_{l, \text { full }}$ has a central/noncentral chi-square distribution with two degrees of freedom depending on whether the signal is present or not. Denoting the threshold as $\gamma$, the probability that the hypothesis variable exceeds $\gamma$ is [13]

$$
P_{l, f u l l}=\int_{\gamma / \sigma_{l, f u l l}^{2}}^{\infty} e^{-\left(x+\mu_{l, f u l l}\right)} I_{0}\left(2 \sqrt{x \mu_{l, f u l l}}\right) d x
$$

where $\mu_{l, \text { full }}=E_{l, f u l l}^{2} / \sigma_{l, \text { full }}^{2}$ and $I_{0}$ is zeroth order modified Bessel function of the first kind. Since the correct acquisition state is $0, P_{0, \text { full }}$ is the detection probability and $P_{l, \text { full }}\left(l=1, \ldots, L_{1}\right)$ are false alarm probabilities.

\subsection{Hypothesis Testing in a Channelized Receiver}

The search states are labeled by $l=0,1, \ldots, L_{2}$. The $l=0$ state corresponds to the correct acquisition state.
In the channelized receiver, $N_{w} / M$ samples are collected at a rate of $1 /\left(M T_{e}\right)$ for each pulse. The search increment step for time offset $T$ is $M T_{e}$ instead of $T_{e}$ as in the fullband receiver. Because of the increased search increment step, the number of channelized receiver search states is reduced by $M$ compared to a fullband receiver, that is, $L_{1}=M L_{2}$.

The ADC samples in the $m$ th subband of the windowed $k$ th pulse is represented in vector form as $e^{j \theta_{l, k, m}}\left(\mathbf{s}_{l, k, m}+\mathbf{n}_{l, k, m}\right)$, where $\mathbf{s}_{l, k, m}$ is the signal vector, $\mathbf{n}_{l, k, m}$ is the noise vector and $\theta_{l, k, m}$ is the $m$ th subband mixer phase. After correlating with $m$ th subband of the windowed $(k+1)$ st pulse then summing all the subband correlation signals, the hypothesis variable of the channelized receiver is

$$
J_{l, c h}=\sum_{m=0}^{M-1}\left|\left(\mathbf{s}_{l, k, m}+\mathbf{n}_{l, k, m}\right)^{H}\left(\mathbf{s}_{l,(k+1), m}+\mathbf{n}_{l,(k+1), m}\right)\right|^{2}
$$

As in the full-band receiver, the hypothesis variable $J_{l, c h}$ is compared with a threshold to determine whether the signal is present. Assuming that the noise among the subbands is uncorrelated, $J_{l, c h}$ becomes the summation of $M$ independent and chi-square distributed random variables. The probability density function (PDF) of $J_{l, c h}$ is the convolution of $M$ chi-square PDFs, each of which corresponds to the squared correlation signal in a subband. The false alarm and detection probabilities, $P_{l, c h}\left(l=1, \ldots, L_{2}\right)$ and $P_{0, c h}$, can then be readily obtained by integrating the PDF of $J_{l, c h}$.

\subsection{Acquisition Performance Comparison}

Following the similar steps as in [13], the singledwell serial-search mean acquisition time of a fullband receiver in terms of $T$ is

$$
\begin{aligned}
T_{a c q, f u l l}= & \frac{2 L_{1}-\left(L_{1}-1\right) P_{0, \text { full }}}{2 P_{0, \text { full }}} \\
& +\frac{\kappa\left(1-P_{0, \text { full }}\right) \sum_{l=1}^{L_{1}} P_{l, \text { full }}}{P_{0, \text { full }}} \\
& +\frac{\kappa \sum_{l=1}^{L_{1}} l P_{l, \text { full }}}{L_{1}}
\end{aligned}
$$

where $\kappa$ is false alarm penalty. In the channelized receiver, the mean acquisition time is similar to (12) ex- 


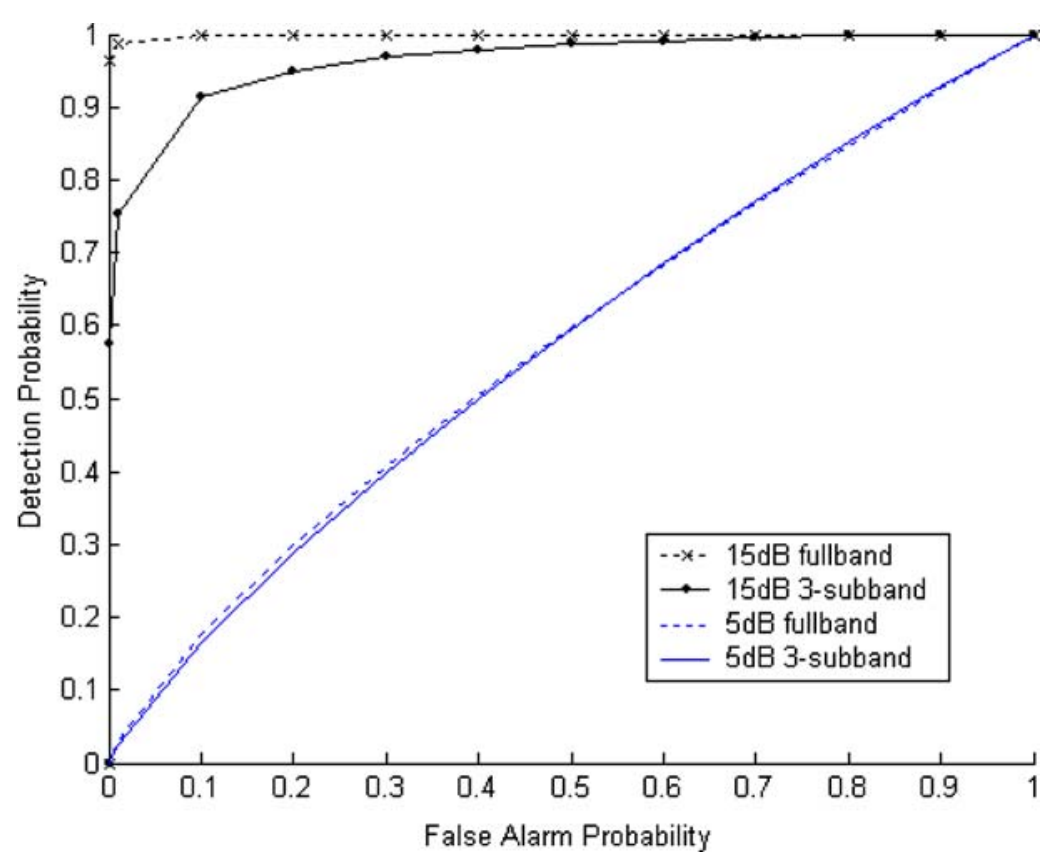

Figure 8. The operating characteristic of the decision device.

cept that $P_{l, \text { full }}\left(l=0, \ldots, L_{2}\right)$ and $L_{1}$ are replaced by $P_{l, c h}$ and $L_{2}$, respectively.

To understand the operating characteristic of the decision device in both receivers, the detection probability $\mathrm{P}_{\mathrm{D}}$, and the false alarm probability when signal is absent in both pulses $\mathrm{P}_{\mathrm{F}}$ are plotted in Fig. 8. The received $E_{b} / N_{0}=5 d B$ or $15 d B$. The channelized receiver has three sub-bands. For a given $\mathrm{P}_{\mathrm{F}}$, the $\mathrm{P}_{\mathrm{D}}$ of the full-band receiver is higher than that of the channelized receiver, because of the loss due to the squaring and summing of the subband signals.

Although $\mathrm{P}_{\mathrm{D}}$ is lower for a given $\mathrm{P}_{\mathrm{F}}$, an important advantage of the frequency channelized receiver is that the number of bins to search is reduced by approximately $M$ as explained earlier. The net result of these two contradictory effects is that the ratio of the mean acquisition time between the channelized and the fullband receivers depends on number of subbands and $E_{b} / N_{0}$. Using (12), the acquisition time ratios at $E_{b} / N_{0}$ of $5 \mathrm{~dB}$ and $15 \mathrm{~dB}$ are plotted in Fig. 9 as a function of number of subbands for a CM1 channel. To compute the acquisition time, we assume that the penalty associated with a false alarm is five time units, i.e., $\kappa=5$. The mean acquisition time is shorter in a channelized receiver than in a fullband receiver due to the reduced search space. As the number of subbands increases, however, the reduction in mean acquisition time suf- fers from diminishing returns because of the increase in false alarm probability.

\section{Fine Synchronization and Detection}

After initial acquisition, the receiver enters the fine synchronization mode, where the timing adjustment feedback loop is initiated to reduce the timing error. The loop, which operates at the symbol rate, adjusts the sampling times (using digital interpolators) to synchronize with the transmitter. Once synchronization is achieved, channel estimation can be performed by averaging the reference pulses. Correlation length can be updated by calculating the signal power in the time bins before and after the current correlation window. The digital receiver structure is shown in Fig. 10. The phase rotation caused by incommensurate clocks can be estimated and compensated $\left(e^{-j \hat{\theta}_{k m}}\right.$ term in the figure) based on the timing loop parameters since timing and phase have a constant relationship.

\subsection{Timing Adjustment Loop}

The transmitted data symbol period $T$ and the receiver clock period $T_{\mathrm{r}}=T+\varepsilon$ are incommensurate due to the free running oscillators in the transmitter and the receiver. After initial acquisition, the timing adjustment 


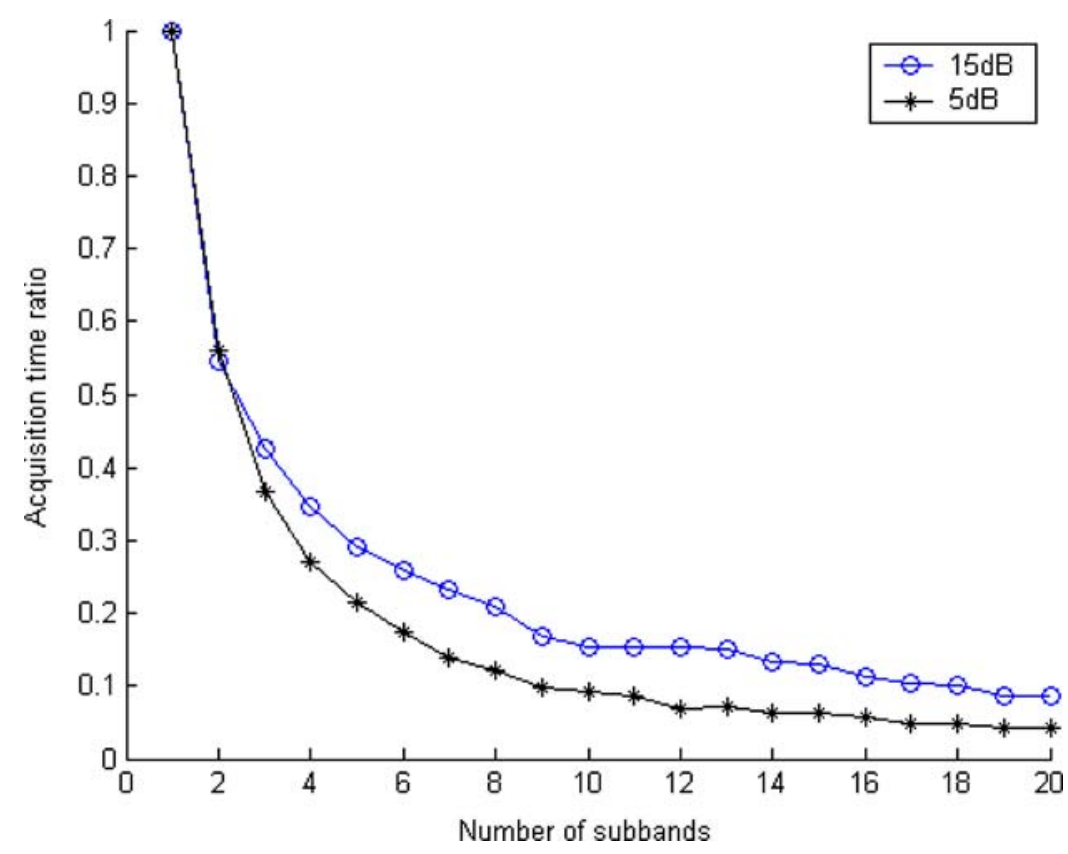

Figure 9. Acquisition time ratio between channelized receivers and a fullband receiver.

loop compensates the remaining timing offset by shifting all samples in a frame by an integer period of $\alpha$ as well as a fractional period of $\mu .(\alpha+\mu) M T_{s}$ is the timing offset with respect to the first pulse. $\mu$ is estimated by the M\&M TED, loop filter and NCO [10].

When 1-bit ADCs are employed to simplify the receiver complexity, the interpolator generates a multibit output, since 1-bit can not represent samples at the fractional period. The multibit output is in the form of two's complement. The pulse correlation function of the 1-bit, 2-bit and 3-bit interpolator output are shown in Fig. 11. They have timing accuracy of 1 , $1 / 2$ and $1 / 6$ of sampling period, respectively. The $3-$ bit output interpolator is adopted to achieve the timing accuracy of $1 / 6$ sampling period. Since the input to the interpolator is 1-bit data, a linear interpolator is adequate. Due to the limited timing accuracy, the linear interpolator is implemented as a combinational logic with two 1-bit sample inputs and one 3-bit timing input.

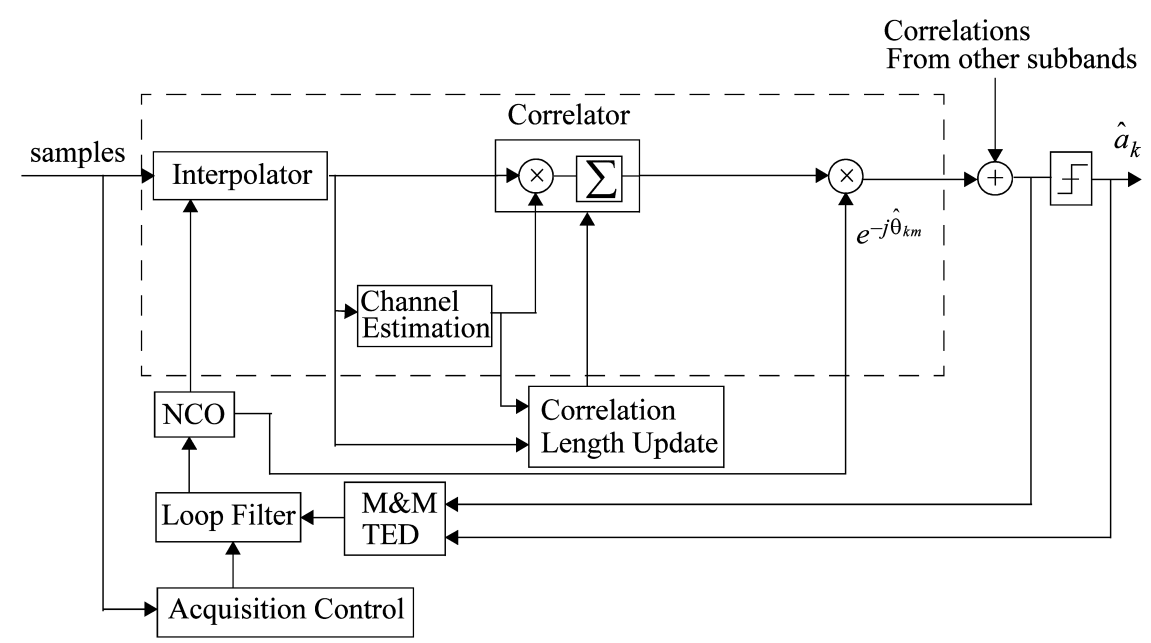

Figure 10. Digital receiver structure. 


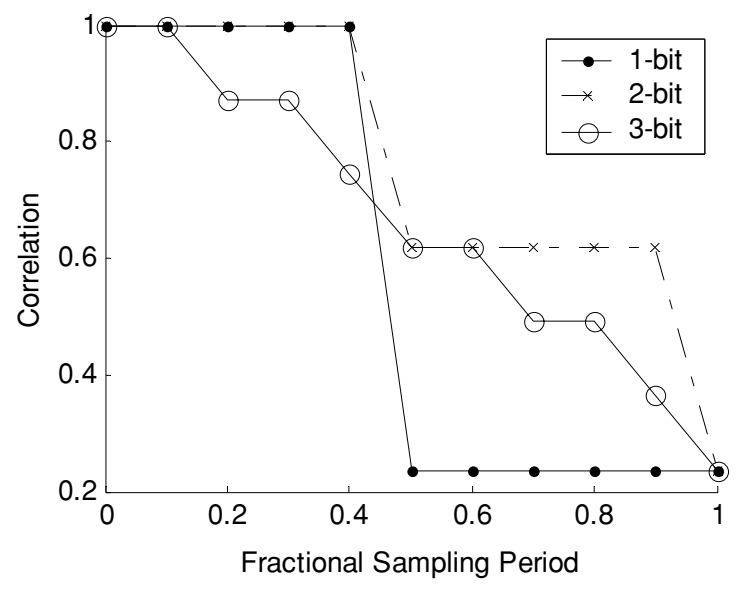

Figure 11. The interpolator output correlation.

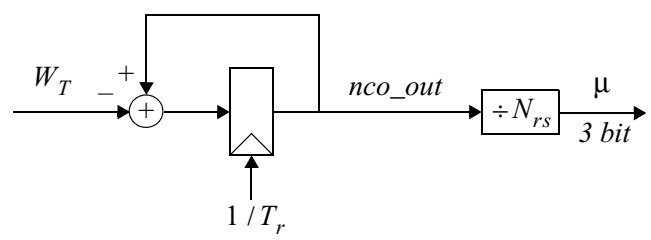

Figure 12. NCO in timing adjustment loop.
A loop filter is used to update the control word of the timing loop $\mathrm{NCO} W_{T}$. In the NCO, the control word determines the ratio between the transmitter symbol period $T$ and the receiver symbol period $T_{r}$

$$
W_{T}=\frac{T}{T_{r}}
$$

which is typically a value close to an integer. A functional block diagram of the NCO is shown in Fig. 12. The NCO register nco_out is updated each $T_{r}$. nco_out represents the required timing shift in terms of $T_{r}$. Since the interpolator needs timing information in terms of $T_{s}$, nco_out is divided by a known integer $N_{r s}$ to produce $\alpha$ and $\mu . N_{r s}$ is defined as $N_{r s}=T_{r} / T_{s}$. By selecting an appropriate $N_{r s}$, the division could be simplified to a shift. Due to the high sampling rate and low duty cycle pulse, $N_{r s}$ can be very large. Although not shown, a 20-bit NCO register and a 3-bit $\mu$ for interpolation achieves near optimal performance.

\subsection{Length of the Correlation Window}

The correlation length needs to be dynamically updated depending on the UWB channel. If the length is too

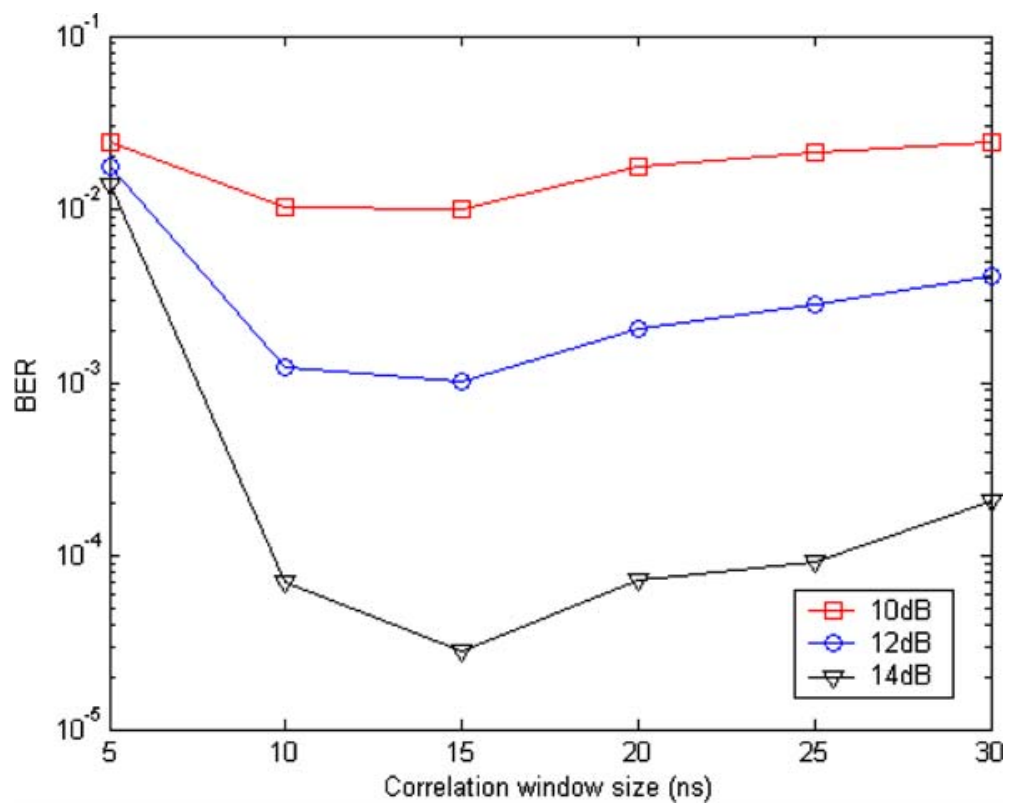

Figure 13. Correlation length effects for CM1 channels. 
short, the receiver is not collecting sufficient signal energy. If the length is too long, extra noise is introduced. The correlation length effects on BER for CM1 channel are plotted in Fig. 13. The simulation is performed with 1-bit $\mathrm{ADC}$ at $E_{b} / N_{0}$ of $10 \mathrm{~dB}, 12 \mathrm{~dB}$ and $14 \mathrm{~dB}$. The correlation window is selected so to minimize BER. The optimum window size for the CM1 channel is in the $10 \mathrm{~ns}$ to $20 \mathrm{~ns}$ range. To achieve good performance for different channels, the length of the correlation window needs to be dynamically updated according to the channel conditions. A 5ns window step appears adequate.

To update the correlation length, the partial correlation values in two extra bins are calculated. One bin is before the correlation window and the other bin is after the window. The correlations of the two bins together with those of the first and the last bins of the correlation window are compared with a threshold (i.e. $50 \%$ of the average bin correlation in the current correlation window). A bin with correlation larger than the threshold after multiple frames should remain or be added to the correlation window. A bin with correlation smaller than the threshold should be removed from the correlation window.

\section{Conclusions}

The baseband processor design of a channelized receiver for UWB TR systems is investigated in this paper. The channelized receiver achieves near optimal performance for small ADC oversampling ratio despite reasonably large variations in analog filter bandwidth. The acquisition performance of the channelized receiver is superior to that of a fullband receiver because of the reduced search space of the channelized receiver. Fine synchronization is implemented with linear interpolators and digital NCOs. The effects of correlation window length are also investigated by simulation.

\section{Acknowledgments}

This work was supported in part by the Army Research Office under contract number DAAD19-01-1-0477 and National Science Foundation under contract number ECS-0134629.

\section{References}

1. V.S. Somayazulu, J.R. Foerster, S. Roy, "Design challenges for very high data rate UWB systems," Signals, Systems and Computers, 2002, vol. 1, pp. 717-721, Nov. 2002.

2. W. Namgoong, "A Channelized Digital Ultra-Wideband Receiver," IEEE Trans. Wireless Comm., vol. 2, pp. 502-510, May 2003.

3. A. Gilloire and M. Vetterli, "Adaptive Filtering in Subbands with Critical Sampling: Analysis, Experiments, and Application to Acoustic Echo Cancellation," IEEE Trans. on Signal Proc. vol. 40, NO. 8, Aug. 1992.

4. R. Hoctor and H. Tomlison, "An Overview of Delay-Hopped, Transmitted-Reference RF Communications," GE Research and Development Center, Tech. Rep. 2001CRD198, 2002.

5. Y. Chao and R. A. Scholtz, "Optimal and suboptimal receivers for ultra-wideband transmitted reference systems," GLOBECOM '03, vol. 2, pp. 759-763, Dec. 2003.

6. H. Kim, D. Park and Y. Joo, "All-digital low-power CMOS pulse generator for UWB system," IEE Electronics Letters, vol. 40, NO. 24, Nov. 2004.

7. B. Parr, etc. "A Novel Ultra-Wideband Pulse Design Algorithm," IEEE Comm. Letters, vol. 7, NO. 5, May 2003.

8. L. Feng and W. Namgoong, "An Oversampled Channelized UWB Receiver," International Workshop on UWBST \& IWUWBS 2004, pp. 410-414, May 2004.

9. L. Feng and W. Namgoong, "Oversampled Channelized Receiver for Transmitted Reference UWB System in the Presence of Narrowband Interference," IEEE Workshop on Signal Processing Systems (SIPS) 2004, pp. 48-52, Oct. 2004.

10. F. M. Gardner, "Interpolation in Digital Modems - Part 1: Fundamentals," IEEE Trans. on Communications, vol. 41, NO. 3, March 1993.

11. E.A. Homier and R.A. Scholtz, "Rapid Acquisition of Ultrawideband Signals in the Dense Multipath Channel," Ultra Wideband Systems and Technologies (UWBST) 2002, pp. 105109, May 2002.

12. R. Blazquez, P. Newaskar and A. Chandrakasan, "Coarse Acquisition for Ultra Wideband Digital Receivers," ICASSP 2003, vol. 4, pp. IV - 137-140, April 2003.

13. K. SH. Zigangirov, "Theory of Code Division Multiple Access Communication,” John Wiley \& Sons, Inc. 2004.

14. K. M.Chugg and M. Zhu, "A new approach to rapid PN code acquisition using iterative message passing techniques," IEEE Journal on Selected Areas in Communications, vol. 23, Issue 5, pp. 884-897, May 2005.

15. L. Reggiani and G. M.Maggio, "Rapid search algorithms for code acquisition in UWB impulse radio communications," IEEE Journal on Selected Areas in Communications, vol. 23, Issue 5, pp. 898-908, May 2005.

16. L. Yang, Z. Tian and G. B.Giannakis, "Non-data aided timing acquisition of ultra-wideband transmissions using cyclostationarity," ICASSP 2003, vol. 4, pp. IV - 121-124, April 2003.

17. S. Franz and U. Mitra, "Integration interval optimization and performance analysis for UWB transmitted reference systems," Joint UWBST \& IWUWBS. 2004, pp. 26-30, May 2004. 


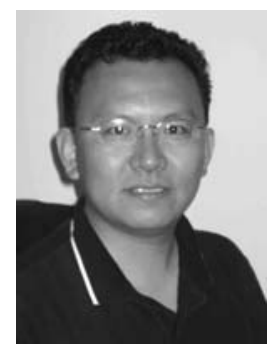

Lei Feng received the B.S. and M.S. degree in electrical engineering from Peking University, Beijing, in 1997 and 2000, respectively. $\mathrm{He}$ is currently working toward the Ph.D degree in electrical engineering at University of Southern California, Los Angeles, CA. His doctoral research focuses on the design of wideband communication transceivers for wireless and wireline applications.

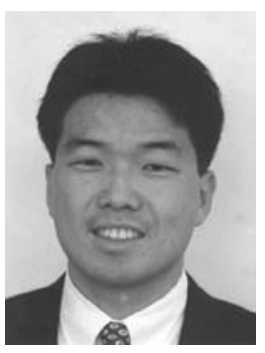

Won Namgoong received the BS degree in Electrical Engineering and Computer Science from the University of California at Berkeley in 1993, and the MS and Ph.D. degrees in Electrical Engineering from Stanford University in 1995 and 1999, respectively. In 1999, he joined the faculty of the Electrical Engineering Department at the University of Southern California, where he is an Assistant Professor. His current research areas include wireless/wireline communication systems, signal processing systems, RF circuits, and lowpower/high-speed circuits. In 2002, he received the National Science Foundation (NSF) CAREER Award. 